\title{
Concepções de enfermeiras egressas da residência acerca da interdisciplinaridade em Centros de Atenção Psicossocial
}

\author{
Conceptions of interdisciplinarity in Psychosocial Care Centers among nurses who completed \\ residency
}

\author{
Concepciones de enfermeras egresadas de la residencia sobre la interdisciplinariedad en Centros de \\ Atención Psicosocial
}

\author{
Caroline Silva Batista Alves' $\odot$, Tiago Braga do Espirito Santo' $\odot$, Edna Gurgel Casanoval $\odot$
}

'Universidade do Estado do Rio de Janeiro, Rio de Janeiro, RJ, Brasil

\begin{abstract}
RESUMO
Objetivo: analisar as concepções das egressas da Residência de Enfermagem Psiquiátrica e Saúde Mental sobre o trabalho interdisciplinar em Centros de Atenção Psicossocial. Método: estudo qualitativo-exploratório. Os dados foram coletados por entrevistas semiestruturadas com quatro participantes, entre julho a agosto de 2017. Utilizou-se a análise temática de Bardin. Aprovado pelo Comitê de Ética em Pesquisa. Resultados: Evidenciou-se o valor da integralidade do cuidado, produzida pelo compartilhamento de saberes. Destacam-se, contudo, os desafios atrelados à hierarquização, à valorização das competências profissionais específicas e à necessária revisão do processo formativo. Conclusão: A interdisciplinaridade é compreendida como fundamental para a integralidade do cuidado, mas vem encontrando dificuldades nos serviços e em seu desenvolvimento na formação lato sensu, devido a preservação do modelo biomédico. Aponta-se para a necessidade de implementação de espaços de educação permanente e revisão curricular dos cursos da saúde, produzindo reflexões que extrapolem a especificidade profissional. Descritores: Serviços Comunitários de Saúde Mental; Práticas Interdisciplinares; Capacitação de Recursos Humanos em Saúde; Enfermagem.
\end{abstract}

\begin{abstract}
Objective: to analyze conceptions of interdisciplinary work in Psychosocial Care Centers among graduates from a Psychiatric Nursing and Mental Health Residency. Method: in this exploratory qualitative study, data were collected by semi-structured interviews of four participants, between July and August 2017. Bardin's thematic analysis was used. Approved by the Research Ethics Committee. Results: the study evidenced the value of comprehensive care produced by knowledge sharing, while highlighting the challenges posed by hierarchization, recognition for specific professional skills and the need for review of the training process. Conclusion: Interdisciplinarity is understood to be fundamental to comprehensive care, but has been encountering difficulties in services and in lato sensu training, due to perpetuation of the biomedical model. It points to the need to implement continued professional development and review of health course curricula, to prompt thinking that goes beyond the specifics of the profession.
\end{abstract}

Descriptors: Community Mental Health Services; Interdisciplinary Placement; Health Human Resource Training; Nursing.

\section{RESUMEN}

Objetivo: analizar las concepciones de los egresados de la Residencia de Enfermería en Psiquiatría y Salud Mental sobre el trabajo interdisciplinario en Centros de Atención Psicosocial. Método: estudio cualitativo-exploratorio. Los datos fueron recolectados a través de entrevistas semiestructuradas con 04 participantes, entre julio y agosto de 2017. Se utilizó el análisis temático de Bardin. Fue aprobado por el Comité de Ética en Investigación. Resultados: Se evidenció el valor de la integralidad de la atención, producida por el intercambio de conocimientos. No obstante, destacan los retos vinculados a la jerarquización, la valoración de las competencias profesionales específicas y a la necesaria revisión del proceso formativo. Conclusión: La interdisciplinariedad se entiende como fundamental para la integralidad de la atención, pero ha estado encontrando dificultades en los servicios y en su desarrollo en la formación lato sensu, debido a la preservación del modelo biomédico. Señala la necesidad de implementación espacios de educación permanente y de revisión curricular de los cursos de salud, produciendo reflexiones que vayan más allá de la especificidad profesional.

Descriptores: Servicios Comunitarios de Salud Mental; Prácticas Interdisciplinarias; Capacitación de Recursos Humanos en Salud; Enfermería.

\section{INTRODUÇÃO}

A Reforma Psiquiátrica Brasileira (RP), iniciada no final da década de 1970, promoveu avanços no modelo de atenção à saúde mental e incentivou a expansão dos serviços extra hospitalares com a Lei n 10.216 de 06 de abril de $2001^{1,2}$. Dez anos depois, foi estabelecida a Rede de Atenção Psicossocial (RAPS), por meio da Portaria no 3.088 , de 23 de dezembro de 2011, que buscou articular e integrar os pontos de atenção para assegurar a integralidade do cuidado. Destaca-se, na referida Portaria, a interdisciplinaridade como estratégia para a promoção da autonomia e inserção social dos usuários ${ }^{3,4}$. 
Desde a sua implementação, a RAPS vem sofrendo ataques, destacando-se o retrocesso apresentado pela Portaria no 3588/2017 e pela Nota Técnica n¹1/2019, que reintroduziram na rede os hospitais psiquiátricos. De todo modo, pode-se, ainda, afirmar que a RAPS consiste em: rede básica, enfermarias psiquiátricas em hospitais gerais, estratégias de desinstitucionalização e de reabilitação psicossocial e serviços especializados de base comunitária ${ }^{3-5}$

Os Centros de Atenção Psicossocial (CAPS) são os serviços especializados e estratégicos para a implantação da reorganização da assistência psiquiátrica, promovendo o cuidado integral e a reabilitação psicossocial dos usuários. Para tanto, necessita-se do trabalho em equipe, tendo a enfermagem importante papel na produção do cuidado ${ }^{6}$. 0 fazer da enfermagem é reconhecido nacional e internacionalmente por melhorar o acesso e a qualidade na saúde mental, mas apresenta grandes desafios à sua prática profissional ${ }^{7}$.

Para este processo de trabalho, destaca-se a interdisciplinaridade como a configuração de equipe que demanda o entendimento de uma problemática comum, exigindo o trabalho conjunto, a aprendizagem mútua e a combinação de elementos de cada categoria ${ }^{8}$. Neste sentido, ela se concretiza pela integração e articulação de diferentes conhecimentos e práticas, voltadas à construção de intervenções em comum, valorizando o saber e as intervenções das categorias profissionais envolvidas ${ }^{9}$.

A interdisciplinaridade é, portanto, fundamental para a reformulação da atuação da enfermagem em saúde mental, por convocar a abertura às novas contingências dos sujeitos e suas singularidades, demandando a superação do pensamento simplista dos processos de saúde e doença, produzindo novos conhecimentos ${ }^{8}$. Nessa perspectiva, os profissionais devem também estar abertos às vicissitudes e ao desejo de superar os caminhos já trilhados, a partir do compartilhamento de novos enfoques.

Esta configuração é relevante para os processos de trabalhos nos CAPS, no qual os profissionais devem ser capazes de articular conhecimentos específicos junto a rede de cuidado ${ }^{10}$.

Nessa conjuntura, a enfermagem, com o intuito de acompanhar as transições no modelo de atenção à saúde mental, vem enfrentando o desafio de reaprender a cuidar, alinhando-se às propostas enfatizadas pela RP e a interdisciplinaridade. Deve-se, neste processo de reconfiguração do cuidado, olhar para as práticas cotidianas da enfermagem nos serviços de saúde mental com o objetivo de construir um novo modelo de cuidado que integre as diversas disciplinas, possibilitando o alcance do cuidado integral e interdisciplinar ${ }^{11}$.

A importância da categoria para a transformação dos processos de trabalho é ressaltada pelo seu grande quantitativo nas equipes de equipes de saúde mental. Somado a isso, conforme deliberação do Conselho Federal de Enfermagem (COFEN), os enfermeiros para trabalharem em saúde mental precisam ter especialização na área. Desta forma, o que se vislumbra é a relevância da força de trabalho composta por enfermeiros especialistas em Saúde Mental ${ }^{12}$.

Tendo em vista que o curso de Pós-Graduação Latu sensu é o espaço de formação necessário para se obter a referida especialização demandada pelo COFEN, é importante investigar como os egressos de um curso de residência em enfermagem psiquiátrica e em saúde mental compreendem o trabalho interdisciplinar nos CAPS.

Desta forma, indagamos: Como estes egressos têm vivenciado a prática interdisciplinar no cotidiano do trabalho em saúde mental? Como a interdisciplinaridade foi abordada na especialização?

Para tanto, tem-se como objetivo analisar as concepções dos egressos da Residência de Enfermagem Psiquiátrica e em Saúde Mental sobre o trabalho interdisciplinar em Centros de Atenção Psicossocial.

\section{MÉTODO}

Trata-se de um estudo qualitativo-exploratório ${ }^{13}$, realizado com quatro enfermeiras que atuam em CAPS de diversas modalidades, egressas de um programa de Residência Uniprofissional de Enfermagem Psiquiátrica e em Saúde Mental, de um Hospital Universitário no Rio de Janeiro/RJ. O referido programa tem duração de dois anos, demandando dedicação exclusiva e remuneração mensal através de bolsa de estudo. A entrada se dá por meio de concurso público, aprovando até três residentes por seleção. A carga horária é dividida entre atividades teóricas (gerais e específicas) e práticas, tendo como cenários de práticas: enfermaria psiquiátrica de um Hospital Geral Universitário, ambulatório de psiquiatria, CAPSII e ambulatório de cardiologia, com consultas em Saúde Mental.

Para o corpus documental, buscou-se nos arquivos da coordenação do programa os contatos das enfermeiras egressas da residência entre os anos de 2006 a 2016.

Durante a delimitação temporal estabelecida, 27 enfermeiras passaram pela Residência em Enfermagem em Psiquiatria e Saúde Mental. Destas, 21 concluíram o curso (seis foram desligadas). Tendo em vista a deficiência dos registros na coordenação do programa, não foi possível localizar o contato com oito egressas. As 13 enfermeiras egressas foram recrutadas através de contato telefônico, atendendo aos seguintes critérios: atuar em CAPS do município do Rio de Janeiro e estar com registro ativo no Conselho Regional de Enfermagem. Apenas cinco egressas 
atenderam aos critérios de inclusão. No entanto houve uma egressa que desistiu da participação na pesquisa, restante o corpus documental de quatro entrevistas.

As entrevistas foram agendadas conforme disponibilidade das participantes e, após a assinatura do Termo de Consentimento Livre e Esclarecido, realizadas nos locais de trabalho, de forma individual, no período de julho a agosto de 2017. Os dados foram coletados a partir de um roteiro de entrevista semiestruturado composto pela caracterização das participantes: idade, sexo, cor, estado civil, número de dependentes, ano de conclusão da residência, local de trabalho e questões relacionadas à trajetória de trabalho no CAPS e à formação na residência. No que diz respeito ao trabalho interdisciplinar, foi questionado sobre a concepção da interdisciplinaridade nos serviços aonde atuavam e como o tema foi abordado durante a formação.

As entrevistas foram gravadas, transcritas e arquivadas. Suas identidades foram preservadas, sendo denominadas por atributos pessoais, escolhidos por elas e enumeradas pela ordem cronológica das coletas de dados, com a seguinte codificação: Liderança1 (L1), Gerencial2 (G2), Curiosa3 (C3) e Sensibilidade4 (S4). Os dados, analisados segundo a Análise de Conteúdo Temática de Bardin ${ }^{13}$, foram organizados para exploração do material, tratamento dos resultados, inferência e interpretação.

A pesquisa seguiu os preceitos éticos preconizados pela Resolução do Conselho Nacional de Saúde no 466 , de 12 de dezembro de 2012, tendo sido aprovada pelo Comitê de Ética em Pesquisa em 26 de junho de 2017.

\section{RESULTADOS E DISCUSSÃo}

As enfermeiras participantes eram mulheres que se autodeclararam brancas, solteiras e sem dependentes, com idade média de 31,75 anos, sendo a menor idade de 29 anos e a maior 34 anos. Três graduaram-se em universidades públicas e uma em faculdade privada. Todas as participantes concluíram a residência em enfermagem psiquiátrica e em saúde mental entre os anos de 2014 a 2016. Quanto à trajetória profissional, todas eram trabalhadoras de CAPS III, sendo a média do tempo de trabalho de dois anos e cinco meses. As enfermeiras declararam ter estes cenários como desejo profissional e, para tanto, participaram de processos seletivos específicos para tais unidades. Apenas uma enfermeira atuava em dois CAPS.

Os depoimentos abordaram o trabalho das enfermeiras no CAPS voltados às práticas gerenciais e assistenciais, sendo estas últimas referentes ao cuidado do corpo, ao acolhimento aos usuários e a atividades na RAPS. O trabalho territorial destacou-se como aquele que, a partir da singularidade do sujeito, viabiliza a produção de saúde e cidadania. Por sua vez, à função gerencial atribuiu-se a provisão de medicamentos e suprimentos, o gerenciamento da equipe de enfermagem e a articulação com a rede de saúde.

A análise dos dados fez emergir duas categorias de análise "O trabalho interdisciplinar e a Enfermagem no contexto do CAPS" e "O desafio da interdisciplinaridade na formação de enfermeiros especialistas em saúde mental".

\section{0 trabalho interdisciplinar e a enfermagem no contexto do CAPS}

As enfermeiras trouxeram em suas falas que a dinâmica do trabalho interdisciplinar é importante para a produção do cuidado no CAPS, pois agrega conhecimentos para enfrentar a complexidade da saúde mental. Evidencia-se nas falas que apenas uma profissão não contempla as múltiplas demandas dos usuários, apontando para a interdisciplinaridade possibilidade de efetivação da integralidade do cuidado, conforme destacado nas falas a seguir:

São casos muito complexos e que um único saber não dá conta de resolver (L1).

Eu avalio que é essencial o trabalho interdisciplinar [...] porque quando a gente vai discutir uma situação, um caso, a gente tem uma visão de cada pessoa, de categoria, de cada formação, acho que a gente não pode se restringir somente a um olhar (S4).

Os depoimentos destacaram a potência da produção do cuidado quando a equipe intervém nos casos de maneira coletiva, mobilizada pelo compartilhamento de saberes. Nesse sentido, o trabalho interdisciplinar ocorre quando os membros da equipe de saúde mental compartilham seus conhecimentos específicos, a fim de completarem-se na produção do cuidado e melhorar a qualidade da atenção. Nesse contexto, a interdisciplinaridade media saberes profissionais para a produção do cuidado pautado na coletividade, evidenciando a importância de ultrapassar as fronteiras de cada categoria, porém conservando aspectos inerentes a cada profissão ${ }^{14}$.

Como potencialidades do trabalho interdisciplinar emergiram das falas das enfermeiras a qualidade do cuidado, que abrange as necessidades de saúde dos usuários. Fomenta-se, portanto, o diálogo na equipe e a articulação das tecnologias de cuidados, otimizando a integralidade da atenção, o que pode ser visualizado nos depoimentos a seguir:

É uma equipe que por mais atravessamentos que tenha, [...] consegue conversar entre si e viabilizar o cuidado, que é o mais importante (C3). 
A gente precisa da visão dos outros também na construção do que a gente acredita que vai ser melhor e junto com eles (usuários), eles também junto com a gente como protagonista desse cuidado (S4).

A partir da visão ampliada do cuidado e dos princípios do trabalho em equipe, amplia-se também a troca e a potência dos encontros profissional-profissional e profissional-usuários. Desta forma, destaca-se a necessidade de consolidar a atuação interdisciplinar no trabalho da enfermagem nos CAPS, de modo que os profissionais possam ter a liberdade e a segurança para compor e intervir nos saberes das demais categorias profissionais, compreendendo o seu papel na equipe, por atitudes menos rígidas ou estáticas ${ }^{15}$.

A concepção apresentada pelas participantes acerca da potência do trabalho interdisciplinar é corroborada por estudos que evidenciam as contribuições da enfermagem na produção do cuidado em saúde mental, sobretudo na construção do Projeto Terapêutico Singular (PTS). O PTS é uma tecnologia de cuidado fundamental para o campo da saúde mental, inserida no contexto da interdisciplinaridade para o atendimento das demandas de saúde dos sujeitos a partir da composição entre a equipe, o usuário e a família ${ }^{16,17}$.

Entretanto, a interdisciplinaridade foi também abordada como um elemento desafiador. As entrevistadas indicaram resistência da equipe quanto aos limites profissionais, sentindo-se menos valorizadas. Essas tensões apontam fragilidades na horizontalidade entre os membros da equipe, como citados nas falas a seguir:

Isso era um "desafiaço"... muito desafio porque eu ficava pensando assim: 'Cara, como eu vou fazer? (L1).

Então, a gente fica o tempo todo numa tensão, de que "eu faço mais do que você" ou "você faz mais do que eu", "tem que fazer mais do meu jeito ou do seu jeito" (G2).

Estes dados são corroborados por estudos acerca do processo de trabalho em Centros de Atenção Psicossociais, apontando, para a perpetuação da hegemonia e supervalorização do saber médico como fatores que ainda dificultam o trabalho interdisciplinar na atenção psicossocial. Da mesma forma, são sinalizadas as dificuldades de comunicação entre os profissionais da equipe ${ }^{18}$.

A enfermagem, ao organizar a assistência em saúde com foco nos problemas, risco e potencialidades de indivíduos e coletivos, utiliza-se do Processo de Enfermagem (PE), saber próprio de sua categoria profissional. Entretanto, apesar das similaridades entre o PE e o PTS, quando a enfermagem se depara com a necessidade construir Projetos Terapêuticos, mediados pelo compartilhamento de conhecimento com outras especificidades, encontram-se limitados, influenciando a integralidade da produção do cuidado ${ }^{17}$.

A relação entre as atribuições na equipe e as especificidades formativas vem sendo demonstrada em estudos que apontam que a equipe de enfermagem ainda sofre com uma visão reducionista do seu saber, sendo compreendida apenas como responsável pelos cuidados de higiene e aqueles relacionados à terapia medicamentosa, incluindo a verificação e administração, as avaliações do uso, a efetividade e as orientações aos usuários e familiares. Identificouse também que a enfermagem é convocada para o desenvolvimento do seu núcleo de conhecimento a partir da prevenção de doenças clínicas ${ }^{16}$.

Para a mudança de modelo assistencial em saúde mental, estudos demarcam a necessidade de criar movimentos na produção do cuidado da enfermagem que se desloquem das práticas tradicionalmente exercidas por esta categoria como, por exemplo, a inserção nas ações grupais e as intervenções na comunidade ${ }^{19}$. Entretanto, mesmo sendo um importante espaço para a produção do cuidado nos CAPS, a condução dos grupos de apoio e das oficinas terapêuticas ainda não é vislumbrada pelas equipes como uma competência da enfermagem ${ }^{16}$.

Destarte, as dificuldades de relacionamento e compartilhamento de saberes afetam o fazer da enfermagem, mas produzem também consequências diretas para o processo de produção de cuidados psicossociais. Esses obstáculos têm notoriedade na construção do PTS, que acaba sendo reduzido às atividades que o usuário está inserido ou aos dias que frequenta o CAPS. Percebe-se que, mesmo considerando toda a trajetória da RP, o cotidiano dos serviços ainda preserva elementos pertencentes ao modelo biomédico, que reduz o compartilhamento de saberes, o que, invariavelmente, recai sobre a qualidade dos PTS, dos processos de trabalho e da gestão do CAPS ${ }^{7,14}$.

Para os usuários dos serviços de saúde mental, é de suma importância a presença dos enfermeiros no processo terapêutico. Eles estão inseridos em grupos, atividades e atendimentos individuais, exercendo um papel de comunicador e intermediador nas relações familiares e, principalmente, na relação interpessoal de apoio durante o tratamento. Os usuários reconhecem a enfermagem como uma profissão capaz de prestar acolhimento e criação de vínculos com esses profissionais ${ }^{19}$.

Como potência para a resolubilidade dessa questão, foram valorizadas pelas participantes: as reuniões de equipe, que permitem maior porosidade entre os limites profissionais e os aspectos específicos de cada área; a importância da organização do trabalho; e a redução das hierarquias entre gerência e trabalhador e estes e os demais. 
As reuniões de equipe são citadas em outros estudos como um espaço propício para a construção coletiva dos trabalhadores e discussões sobre os casos acompanhados, promovendo arranjos organizacionais que conduzem a realização das intervenções da equipe, sendo, portanto, reconhecido como um espaço interdisciplinar. Nas reuniões as interações ocorrem sem que se percam as especificidades, mas olhando para um cuidado ampliado em saúde mental ${ }^{20}$.

Para a realização das atividades nos CAPS, os profissionais contam com as normativas e os procedimentos, sobretudo aqueles dispostos em manuais e portarias do Ministério da Saúde. Entretanto, o que está geralmente descrito é o que/como fazer de modo defasado aos pressupostos da interdisciplinaridade. Depreende-se assim, a necessidade de atualização dos documentos orientadores, na direção de assegurar a interdisciplinaridade.

Desta forma, a análise dos depoimentos permitiu acessar contradições entre o entendimento das enfermeiras e o que é praticado nos cotidianos dos serviços. Se por um lado, as entrevistadas entendem os benefícios de uma prática interdisciplinar na produção de cuidados em saúde mental no CAPS, por outro lado, evidencia-se a dificuldade de efetivação destes pressupostos na relação com a equipe dos serviços, vinculada sobretudo à dificuldade de validação do lugar ocupado pela enfermagem nessa clínica em transformação.

\section{O desafio da interdisciplinaridade na formação de enfermeiros especialistas em saúde mental}

Segundo as participantes do estudo, não foram abordados marcos teóricos-conceituais e práticos do trabalho interdisciplinar em saúde no decorrer da especialização. Persiste, assim, uma formação que preserva a validação dos saberes específicos de cada categoria. Em seus depoimentos pode-se constatar a relação hierárquica, evidenciada pela desigualdade de poderes entre os profissionais, na composição das equipes de saúde.

[...]quem tem uma formação mais antiga, tem ainda muita dificuldade nisso. A gente é formada em casta, formada por hierarquias e isso eu falo não só de formação de graduação, falo de formação social [...] (G2).

[...] tem uma tendência, um empuxo para você fazer o seu papel [...] (L1).

[...] fazer valer a sua opinião frente a opinião do outro (C3).

O que se evidencia é a formação deficitária no que tange às discussões sobre interdisciplinaridade e a reduzida articulação destes com as políticas públicas de Saúde Mental. As enfermeiras entrevistadas relataram dificuldades em apropriar-se de novas ações e de realizarem o trabalho interdisciplinar, atribuindo este impasse à formação restrita ao específico da Enfermagem. Este fato relaciona-se ao estreito contato que as residentes têm nos campos práticos com profissionais de enfermagem que ainda necessitam ressignificar o objeto de cuidado na saúde mental - da doença para o sujeito - e a necessidade de construir esse novo modelo alinhado com a atenção psicossocial ${ }^{21}$.

Estudos corroboram o encontrado no relato das participantes ao evidenciarem que o trabalho interdisciplinar é dificultado pelas formações acadêmicas ofertadas aos profissionais da saúde, que preservam o modelo biomédico. Os conteúdos relacionados à RP ainda carecem de maiores aprofundamentos e melhores abordagens, sobretudo na formação da enfermagem, que ainda prioriza o enfoque biológico e as burocracias em detrimento da integralidade do cuidado 22 .

A falta de clareza quanto às habilidades e competências para a enfermagem em saúde mental na atenção psicossocial também aparece em outros estudos como um importante obstáculo para a produção do cuidado, limitando as contribuições desta categoria ao que foi tradicionalmente desenvolvido pelo modelo biomédico ${ }^{22}$.

Depreende-se assim, a necessidade de oportunizar-se a aprendizagem, teórico e prática, de modo compartilhado com outras categorias profissionais para que o trabalho interdisciplinar se presentifique no cotidiano dos serviços.

Para tanto, faz-se necessária reformas do processo de ensino-aprendizagem e das práticas pautadas no modelo biomédico, na direção de ações interdisciplinares voltadas às trocas e produção de conhecimentos em benefício da ciência e comunidade ${ }^{21}$. Desta forma, depreende-se a necessidade de articulação e atualização dos programas de residência uniprofissional em enfermagem psiquiátrica e em saúde mental aos pressupostos da RP, que rompe com a lógica manicomial e convoca à produção de novos conhecimentos e saberes no cuidado com as pessoas em sofrimento psíquico ${ }^{10}$.

Nessa direção, aponta-se como possibilidade de ressignificação do modelo de atenção em saúde mental a potência encontrada nas residências multiprofissional em saúde, criadas com o objetivo de reorientar a formação por meio da prática compartilhada, que tem como característica a superação das fragilidades das formações por especificidades e, assim, consolidar as diretrizes do SUS ${ }^{17}$.

Tendo em vista os pressupostos da RP, não se pode pensar no trabalho do CAPS, apenas pela presença de um conjunto de especialistas, sem a integração das práticas profissionais. Desta forma, para que haja mudanças significativas em direção ao trabalho colaborativo, os profissionais precisam aprender em suas trajetórias formativas ferramentas relacionais que proporcionem a troca de conhecimentos que redirecionam a produção de cuidados. 


\section{Limitações do estudo}

O estudo apresenta como limitação, a realização da investigação com um reduzido quantitativo de enfermeiras egressas de apenas uma pós-graduação latu senso. Desta forma, cabem novos estudos que ampliem esse universo, expandindo a investigação para outras residências de enfermagem e em outros dispositivos de saúde mental, o que poderá ofertar novas reflexões acerca do tema.

\section{CONCLUSÃO}

O estudo aponta que as enfermeiras entendem a importância do trabalho interdisciplinar no CAPS para a produção de cuidado em saúde mental. Este dado é evidenciado a partir do valor ofertado à integralidade do cuidado ao usuário, produzido por meio de uma maior aproximação e diálogo entre os trabalhadores, em prol do compartilhamento de saberes.

Contudo, evidencia-se também que os desafios colocados para a implementação de um trabalho interdisciplinar se apresentam na relação com a equipe profissional, sobretudo a valorização das especificidades profissionais, destacando-se os resquícios de uma hierarquização, historicamente construída pelo modelo biomédico.

No que tange às suas formações na residência, as enfermeiras entrevistadas revelaram que o trabalho interdisciplinar foi pouco abordado nas suas trajetórias acadêmicas, o que aponta para a necessidade de revisão curricular dos programas de pós-graduação latu senso, da modalidade residência em Enfermagem Psiquiátrica e em Saúde Mental.

Admite-se, portanto, que para a realização de um trabalho de excelência nos CAPS, é preciso repensar a formação uniprofissional, que fragmenta os processos de trabalho, conduzindo à construção de um cuidado pautado pela interdisciplinaridade e reiterando o compromisso com a transformação do modelo de atenção em saúde mental.

Nesse sentido, o tema da interdisciplinaridade apresenta-se, ainda, como um desafio a ser enfrentado no cotidiano dos CAPS e na formação de enfermeiros especialistas em enfermagem psiquiátrica e em saúde mental. É essencial a abordagem desta temática na formação e no cotidiano dos serviços e, portanto, essa perspectiva deve estar fortemente inserira, tanto na educação permanente quanto nas residências, possibilitando mudanças na produção de cuidado em saúde mental.

\section{REFERÊNCIAS}

1. Nunes, $\mathrm{MO}$ et al. Psychiatric reform and counter-reform: an analysis of a socio-political and sanitary crisis at national and regional level. Ciência \& Saúde Coletiva [Internet]. 2019 [cited 2020 Jul 17]; 24(12):4489-98. DOI: https://doi.org/10.1590/1413812320182412.25252019 .

2. Casa Civil (Br). Lei no 10.216, de 06 de abril de 2001. Dispõe sobre a proteção e os direitos das pessoas portadoras de transtornos mentais e redireciona o modelo assistencial em saúde mental. Diário Oficial da União. 2001. [cited 2020 Jul 17]; Available from: http://www.planalto.gov.br/ccivil_03/leis/leis_2001/l10216.htm.

3. Ministério da Saúde (Br). Portaria no 3.088, de 23 de dezembro de 2011. Institui a Rede de Atenção Psicossocial para pessoas com sofrimento ou transtorno mental e com necessidades decorrentes do uso de crack, álcool e outras drogas, no âmbito do Sistema Único de Saúde. Brasília (DF): Ministério da Saúde; 2011. [cited 2020 Jul 17]; Available from: https://hpm.org.br/wpcontent/uploads/2014/09/lei-no-10.216-de-6-de-abril-de-2001.pdf.

4. Silva PO, Silva DVA, Rodrigues CAO et al. Nursing Clinical Care In Mental Health. Rev enferm UFPE [Internet]. 2018 [cited 2021 Apr 16]; 12(11):3133-46. [cited 2020 Jul 17]; Available from: https://pesquisa.bvsalud.org/portal/resource/pt/biblio-998072.

5. Ministério da Saúde (Br). Portaria no 3.588, de 21 de dezembro de 2017. Dispõe sobre a Rede de Atenção Psicossocial, e dá outras providências. Diário Oficial da União. 2017. [cited 2020 Jul 17]; Available from: https://bvsms.saude.gov.br/bvs/saudelegis/gm/2017/prt3588_22_12_2017.html.

6. Ministério da Saúde (Br). Portaria GM n. 336, de 19 de fevereiro de 2002. Estabelece que os Centros de Atenção Psicossocial poderão constituir-se nas seguintes modalidades de serviços: Caps I, Caps II e Caps III, definidos por ordem crescente de porte/complexidade e abrangência populacional, conforme disposto nesta Portaria. Diário Oficial da União. 2002. [cited 2021 Apr 13]; Available from: https://bvsms.saude.gov.br/bvs/saudelegis/gm/2002/prt0336_19_02_2002.html.

7. Phoenix BJ, Hurd M, Chapman SA. Experience of Psychiatric Mental Health Nurse Practitioners in Public Mental Health. Nurs Admin Q [Internet]. 2016 [cited 2021 Apr 13]; 40(3):212-24. DOI: https://doi.org/10.1097/NAQ.0000000000000171.

8. Anjos Filho NC, Souza AMP. The workers' perceptions about the multiprofessional teamwork at a Psychosocial Care Center in Salvador, Bahia, Brazil. Interface (Botucatu) [Internet]. 2017 [cited 2020 Jul 23]; 21(60):63-76. DOI: https://doi.org/10.1590/1807-57622015.0428.

9. Machado AM, Braga, FJL, Maia RLA, Shimba DS, Mardegan GR, Souza BCO, Silva JA. Bandeira Científica Project: an Interdisciplinary Extension Course with Impacts on the Professional Training in Psychology. Psicologia: Ciência e Profissão [Internet]. 2020 [cited 2021 Jul 17] e213952 (40):1-12. DOI: https://doi.org/10.1590/1982-3703003213952.

10. Fernandes RL, Miranda FAN. Analysis of the theory of interpersonal relationships: nursing care in Psychosocial Care Centers. Rev Enferm UFPE online [Internet]. 2016 [cited 2018 Apr 22]; 10(2):880-86. Available from: https://periodicos.ufpe.br/revistas/revistaenfermagem/article/view/11032. 
11. Tisott, ZL et al. Conception of multiprofessional residents on the management of mental health services. Rev. Eletrônica Saúde Mental Álcool Drog. [Internet]. 2018 [cited 2020 Jul 22] 13(3):140-47. DOI: https://doi.org/10.11606/issn.1806-6976.v13i3p140147.

12. Conselho Federal de Enfermagem (Br). Resolução no599 de 19 de dezembro de 2018. Aprova Norma Técnica Para Atuação da Equipe de Enfermagem em Saúde Mental e Psiquiatria. Brasília. Diário Oficial da União. 2018.

13. Bardin L. Análise de conteúdo. Lisboa: Edições 70; 2020.

14. Jafelice GT, Marcolan JF. The multiprofessional work in the Psychosocial Care Centers of São Paulo State. Rev. Bras. Enferm [Internet]. 2018 [cited 2020 Jul 18];71supl. 5:2131-8. DOI: https://doi.org/10.1590/0034-7167-2017-0300.

15. Bezerra EBN, Silva EF, Máximo TACO, Melo, JSVB. The work of interdisciplinary teams in Psychosocial Care Centers (CAPS). Estud. pesqui. psicol. [Internet]. 2018 [cited $2020 \mathrm{Jul} 22$ ]; 18(1):169-88. Available from: http://pepsic.bvsalud.org/scielo.php?script=sci_arttext\&pid=S1808-42812018000100010.

16. Santos EO, Eslabão AD, Kantorski LP, Pinho LB. Nursing practices in a psychological care center. Rev. Bras. Enferm. [Internet]. 2020 [cited 2021 Apr 16]; 73(1):e20180175. DOI: http://dx.doi.org/10.1590/0034-7167-2018-0175.

17. Rocha EN, Lucena AF. Single Therapeutic Project and Nursing Process from an interdisciplinary care perspective. Rev Gaúcha Enferm [Internet]. 2018 [cited 2021 Apr 16]; 39:e2017- 0057. DOI: https://doi.org/10.1590/1983-1447.2018.2017-0057.

18. Almeida MM, Schall VT, Martins AM, Modena CM. Representations of caretakers about attention in schizophrenia. Psicologia em Estudo [Internet]. 2010 [cited 2020 Jul 13]; 41(1):110-7. Available from: https://revistaseletronicas.pucrs.br/ojs/index.php/revistapsico/article/view/5417.

19. Biffi D, Nasi C. Users' expectations about the practice of nurses in a Psychosocial Care Center. Rev. Rene [Internet]. 2016 [cited 2021 Apr 19]; 17(6):789-96. DOI: https://doi.org/10.15253/2175-6783.2016000600009.

20. Pinho ES, Souza ACS, Esperidião E. Working processes of professionals at Psychosocial Care Centers (CAPS): an integrative review. Ciênc. saúde coletiva [Internet]. 2018 [cited 2021 Apr 12]; 23(1):141-52. DOI: https://doi.org/10.1590/141381232018231.08332015.

21. Jafelice GT, Marcolan JF. Perception of mental health professionals about the multiprofessional work with residents. Rev. enferm. UFPE online [Internet]. 2017 [cited 2020 Jul 20]; 11(2):542-50. Available from: https://periodicos.ufpe.br/revistas/revistaenfermagem/article/download/11972/14519.

22. Lopes PF, Garcia APRF, Toledo VP. Nursing process in the everyday life of nurses in Psycho-Social Attention Centers. Rev Rene [Internet]. 2014 [cited 2021 Apr 18]; 15(5):780-8. DOI: https://doi.org/10.15253/2175-6783.2014000500007. 